\title{
Control Clínico en Pacientes Rehabilitados Mediante Prótesis Fija Total con carga Inmediata. Revisión de la Literatura
}

\author{
Clinical Control of Patients Treated with an Immediately Loaded \\ Implant-Supported Prosthesis. A Systematic Review
}

\author{
Emanuel Figueroa Ahumada \& lan Raby Olavarría
}

FIGUEROA, A. E. \& RABY, O. I. Control clínico en pacientes rehabilitados mediante prótesis fija total con carga inmediata. Revisión de la literatura. Int. J. Odontostomat., 12(1):21-28, 2018.

RESUMEN: Son varias las complicaciones que se pueden presentar posterior a la instalación de una prótesis fija total con carga inmediata (PFTCl), estas pudiesen generar desde el daño de la estructura protésica provisoria hasta la pérdida de la oseointegración de los implantes dentales. La escasa evidencia sobre el comportamiento clínico que se debe tener posterior a este tipo de tratamiento indica la necesidad de controlar mejor estas complicaciones. En esta revisión narrativa, el 3 de marzo de 2016 se realizó una búsqueda electrónica en la base de datos PubMed. Para poder efectuar una investigación acabada los filtros de búsqueda fueron ajustados de la siguiente manera. Se utilizaron los términos de búsqueda "immediate loading" AND "full arch" AND "Resonance frequency analysis". Los que fueron sometidos a los criterios de inclusión y exclusión, dando como resultado un total de 15 estudios. Todos los estudios eran ensayos clínicos controlados, donde se encontraron complicaciones como fracturas, desalojos y desgaste de la estructura protésica, entre otros. Se pudo observar que desde el día de la carga hasta un mes después el valor ISQ (cociente de estabilidad del implante) disminuye, para luego aumentar paulatina y progresivamente. En conclusión, no existe evidencia sobre el número y rango de sesiones necesarias para el control clínico de una PFTCI. El momento más indicado para la extracción de la prótesis provisoria e instalación de la prótesis definitiva es durante el $3^{\circ}$ y $4^{\circ}$ mes posterior a la carga inmediata. Existe escasa evidencia sobre el número, momento y tipo de examen imageneológico a solicitar. La presencia de complicaciones protésicas posterior a una PFTCI hace necesaria la confección de un protocolo de control clínico para este tipo de tratamiento.

PALABRAS CLAVE: carga inmediata, arco completo, análisis de frecuencia de resonancia.

\section{INTRODUCCIÓN}

Esposito et al. (2013) en su trabajo sobre protocolos de carga, concluyó que la carga inmediata es un procedimiento que se puede efectuar con un éxito predecible, pero la tendencia indica que los implantes con carga inmediata fallan con más frecuencia que los con carga convencional. Además, se concluyó que la carga inmediata en maxilar inferior está bien documentada, no así en maxilar superior. La literatura sobre carga inmediata con prótesis fija total en maxilar superior muestra resultados exitosos si se sabe utilizar los criterios de selección de pacientes, implantes, tratamiento quirúrgico y protésico.

Los estudios de pacientes rehabilitados con $\mathrm{PFTCl}$ mencionan que las complicaciones, tanto bio- lógicas como protésicas son muy escasas (Peñarrocha-Oltra et al., 2014). Por lo general las complicaciones intraoperatoria son raras, y en el caso de las postoperatorias inmediata las únicas son la hinchazón y el dolor, siendo estas de poca gravedad (Degidi et al., 2005; Kinsel \& Liss, 2007; Weber et al., 2009). Por otra parte, un estudio de van Steenberghe et al. (2005) aclara que durante el proceso de oseointegración se podría producir fractura de la estructura protésica provisoria en una carga inmediata, presentándose de forma frecuente y con un grado de complejidad bajo al momento de querer reparar la estructura (Pieri et al., 2009). Una vez concretada la oseointegración se pueden encontrar complicaciones biológicas, como lo son la perimplantitis y la mucositis 
(van Steenberghe et al.). Otros estudios, enfatizan que las complicaciones más graves que conducen al fracaso de los implantes es la fractura total de la prótesis acrílica producto de fuerzas nocivas (Grunder, 2001; Maló et al., 2012).

Al evaluar la literatura, resalta el hecho de que los pocos estudios en PFTCl disponibles usan diferentes procedimientos quirúrgicos, tipos de prótesis, tiempos de carga y diferentes diseños de investigación (Peñarrocha-Oltra et al.). No obstante, aunque la evidencia científica en PFTCl sea poco concreta y estandarizada, esta técnica es muy utilizada por los clínicos, puesto que tiene una tasa de éxito y sobrevida del implante muy similar a la del protocolo convencional (Horwitz et al., 2007; Sanz et al., 2015).

Teniendo en cuenta lo anterior, y el hecho de que no existen estudios sobre el control clínico en $\mathrm{PFTCl}$, es que nace la necesidad de confeccionar un protocolo clínico que guíe al profesional, para así poder efectuar un análisis y control más acabado de alguna eventualidad o complicación posterior a una carga inmediata, y de esta manera otorgar un mayor confort de tratamiento al paciente.

\section{MATERIAL Y MÉTODO}

En esta revisión bibliográfica de tipo narrativa, se realizó una búsqueda electrónica el 3 de marzo de 2016 en la base de datos PubMed. Para poder efectuar una investigación acabada los filtros de búsqueda fueron ajustados de la siguiente manera.

1. Tipo artículo: Ensayo clínico, revisión sistémica y narrativa.

2. Disponibilidad del texto: Texto completo, texto completo gratis y abstracto.

3. Años de publicación: Desde el año 2000 en adelante.

4. Especie: Humanos.

5. Idioma: Inglés y español.

Una vez aplicados los filtros, se procedió a ingresar los términos de búsqueda: "immediate loading" AND "full arch" AND "Resonance frequency analysis", de forma individual y combinadas. Este procedimiento dio como resultado un total de 106 investigaciones.
Posterior a esto se procedió a aplicar los criterios de inclusión:

1. Carga inmediata en prótesis fija total para maxilar superior, inferior o ambas.

2. Seguimiento de a los menos 6 meses posterior a la carga inmediata.

3. Estudios que mencionen número y rango de sesiones necesarias para el control clínico de la carga inmediata.

4. Estudios que mencionen tipo, número y rango de exámenes imagenológico a solicitar.

5. Estudios que mencionen el momento en que se debe retirar por primera vez la prótesis provisoria.

6. Estudios relacionados con la evaluación de la cicatrización ósea posterior a una carga inmediata.

7. Estudios que mencionen tipos de complicaciones en prótesis fija total con carga inmediata.

Lo anterior arrojo un total de 19 estudios, a los cuales se les aplicaron los criterios de exclusión:

1. Paciente fumadores pesados (+ 10 cigarrillos diarios).

2. Pacientes sistémicamente comprometidos.

3. Paciente con enfermedad periodontal activa.

4. Implantes cigomáticos.

5. Implantes cortos.

6. Implantes de zirconio.

7. Cirugía guiada por computador.

8. CAD-CAM.

9. Torque de inserción del implante menor a 35 newton. Producto de lo anterior, este proceso arrojo un total de 15 estudios que cumplían con los criterio de selección.

\section{RESULTADOS}

Los resultados vistos en los ECR (ensayos clínicos randomizados) que utilizaron protocolo de car- 
ga inmediata con prótesis fija de arco completo varían tanto en el número, tiempo y periodicidad en que citaron a control a cada uno de los pacientes. Podemos ver que en algunas investigaciones, independiente de su objetivo, el primer control fue a la $1^{\circ}$ semana después de la carga inmediata, en otros a los 10 días o incluso entre el $1^{\circ}$ y $2^{\circ}$ mes (Tabla I).

Sobre el retiro de la prótesis provisoria por primera vez, como también la instalación de la prótesis definitiva, se observó que los estudios muestran diferencias en el momento que este procedimiento se lleva a cabo, e incluso en algunos no informan este hecho. El retiro del provisorio va desde los 10 días hasta los 6 meses. La instalación de prótesis definitiva va desde los 3 a 6 meses. En algunos casos algunos estudios no especifican esta información (Tabla II).

En la toma de exámenes imagenológicos, los resultados arrojaron diferencias en el tipo de examen

Tabla I. Estudios con PFTCl y sus diferencias en número, momento y frecuencia de citación a controles.

\begin{tabular}{|c|c|c|c|c|c|c|c|}
\hline Autor & Año & $\begin{array}{l}\text { Tipo de } \\
\text { estudio }\end{array}$ & Análisis & $1^{\circ}$ control & $2^{\circ}$ control & $3^{\circ}$ control & Otros \\
\hline Bergkvist & 2008 & ECC & $\begin{array}{l}\text { Sobrevida de la } \\
\text { carga inmediata. }\end{array}$ & 1 mes & 8 meses & 20 meses & 32 meses \\
\hline Agliardi & 2010 & ECC & $\begin{array}{l}\text { Sobrevida de la } \\
\text { carga inmediata. }\end{array}$ & 1 semana & 3 meses & 6 meses & 9 meses \\
\hline Acocella & 2011 & ECC & $\begin{array}{l}\text { Efectividad de la } \\
\text { carga inmediata. }\end{array}$ & 10 días & 6 meses & 12 meses & - \\
\hline Ganeles & 2001 & ECC & $\begin{array}{l}\text { Efectividad de la } \\
\text { carga inmediata. }\end{array}$ & 1 semana & 2 semanas & 3 semanas & $1 \mathrm{vez}$ al mes \\
\hline Malo & 2011 & ECC & $\begin{array}{l}\text { Sobrevida de la } \\
\text { carga inmediata. }\end{array}$ & $1 \mathrm{mes}$ & 2 meses & 3 meses & $\begin{array}{c}4-6-7-9-10 \\
11-12\end{array}$ \\
\hline Piano & 2015 & ECC & $\begin{array}{l}\text { Efectividad de la } \\
\text { carga inmediata }\end{array}$ & 1 semana & 2 semanas & 3 meses & 12 meses \\
\hline Testori & 2007 & ECC & $\begin{array}{l}\text { Efectividad de la } \\
\text { carga inmediata }\end{array}$ & 1 mes & 3 meses & 6 meses & 12 meses \\
\hline Eccellente & 2011 & ECC & $\begin{array}{l}\text { Efectividad de la } \\
\text { carga inmediata. }\end{array}$ & 2 semanas & 8 semanas & 10 semanas & - \\
\hline
\end{tabular}

-:Ausencia de control o información no especificada en la investigación. PTFCl: Prótesis fija total con carga inmediata.

Tabla II. Estudios con PFTCI y sus diferencias en el momento del retiro de la prótesis provisoria e instalación de la estructura definitiva.

\begin{tabular}{|c|c|c|c|c|c|}
\hline Autor & Año & $\begin{array}{l}\text { Tipo de } \\
\text { estudio }\end{array}$ & Análisis & $\begin{array}{l}\text { Retiro de prótesis } \\
\text { provisoria }\end{array}$ & $\begin{array}{l}\text { Instalación } \\
\text { Prótesis definitiva }\end{array}$ \\
\hline Bergkvist & 2008 & ECC & Sobrevida de la carga inmediata. & 10 a 12 días & 15 semanas \\
\hline Agliardi & 2010 & ECC & Sobrevida de la carga inmediata. & 4 a 6 meses & 4 a 6 meses \\
\hline Acocella & 2011 & ECC & Efectividad de la carga inmediata. & 3 meses & - \\
\hline Ganeles & 2001 & ECC & Efectividad de la carga inmediata. & - & 3 a 6 meses \\
\hline Malo & 2011 & ECC & Sobrevida de la carga inmediata. & - & 6 meses \\
\hline Piano & 2015 & ECC & $\begin{array}{l}\text { Efectividad de la carga inmediata } \\
\text { con implantes inclinados. }\end{array}$ & 3 meses & - \\
\hline Testori & 2007 & $\mathrm{ECC}$ & $\begin{array}{l}\text { Efectividad de la carga inmediata } \\
\text { con implantes inclinados. }\end{array}$ & - & 3 meses \\
\hline Eccellente & 2011 & ECC & Efectividad de la carga inmediata. & 2 semanas & 6 meses \\
\hline
\end{tabular}

- : Información no especificada en la investigación. PTFCl: Prótesis fija total con carga inmediata. 
Tabla III. Estudios con PFTCI y sus diferencias en el número, momento y tipo de examen imagenológico a solicitar.

\begin{tabular}{|c|c|c|c|c|c|c|c|}
\hline Autor & Año & $\begin{array}{l}\text { Tipo de } \\
\text { estudio }\end{array}$ & Análisis & $1^{\circ}$ Toma & $2^{\circ}$ Toma & $3^{\circ}$ Toma & Tipo \\
\hline Bergkvist & 2008 & ECC & $\begin{array}{l}\text { Sobrevida de la } \\
\text { carga inmediata. }\end{array}$ & $\begin{array}{c}\text { Inmediatamente } \\
\text { posterior a la carga } \\
\text { inmediata. }\end{array}$ & 8 meses & $\begin{array}{c}20 \text { y } 32 \\
\text { meses }\end{array}$ & Periapical \\
\hline Agliardi & 2010 & ECC & $\begin{array}{l}\text { Sobrevida de la } \\
\text { carga inmediata. }\end{array}$ & 6 meses & $\begin{array}{c}12 \\
\text { meses }\end{array}$ & - & $\begin{array}{l}\text { Panorámica } \\
\text { y periapical }\end{array}$ \\
\hline Eccellente & 2011 & $\mathrm{ECC}$ & $\begin{array}{l}\text { Efectividad de la } \\
\text { carga inmediata. }\end{array}$ & 6 meses & $\begin{array}{c}12 \\
\text { meses }\end{array}$ & - & Panorámica \\
\hline Ganeles & 2001 & ECC & $\begin{array}{l}\text { Efectividad de la } \\
\text { carga inmediata. }\end{array}$ & 3 meses & 6 meses & - & $\begin{array}{l}\text { Panorámica } \\
\text { y periapical }\end{array}$ \\
\hline Malo & 2011 & ECC & $\begin{array}{l}\text { Sobrevida de la } \\
\text { carga inmediata. }\end{array}$ & 6 meses & $\begin{array}{c}12 \\
\text { meses }\end{array}$ & - & $\begin{array}{l}\text { Panorámica } \\
\text { y periapical }\end{array}$ \\
\hline Piano & 2015 & $\mathrm{ECC}$ & $\begin{array}{l}\text { Efectividad de la } \\
\text { carga inmediata } \\
\text { con implantes } \\
\text { inclinados. }\end{array}$ & $\begin{array}{c}\text { Inmediatamente } \\
\text { posterior a la carga } \\
\text { inmediata. }\end{array}$ & $\begin{array}{c}12 \\
\text { meses }\end{array}$ & - & Periapical \\
\hline Testori & 2007 & ECC & $\begin{array}{l}\text { Efectividad de la } \\
\text { carga inmediata } \\
\text { con implantes } \\
\text { inclinados. }\end{array}$ & 1 mes & 3 meses & 6 meses & Periapical \\
\hline Acocella & 2011 & ECC & $\begin{array}{l}\text { Efectividad de la } \\
\text { carga inmediata. }\end{array}$ & - & - & - & - \\
\hline
\end{tabular}

- : Ausencia de toma de radiografía o información no especificada en la investigación. PTFCl: Prótesis fija total con carga inmediata.

Tabla IV. Presencia o ausencia de complicaciones protésicas en una prótesis fija total con carga inmediata.

\begin{tabular}{|c|c|c|c|c|c|c|c|c|}
\hline Autor & Año & $\begin{array}{l}\text { Tipo de } \\
\text { estudio }\end{array}$ & Análisis & $\begin{array}{l}\text { Aflojamiento } \\
\text { de tornillo }\end{array}$ & $\begin{array}{l}\text { Fractura } \\
\text { de } \\
\text { tornillo }\end{array}$ & $\begin{array}{l}\text { Fractura } \\
\text { estructura } \\
\text { protésica }\end{array}$ & $\begin{array}{l}\text { Fractura } \\
\text { de diente } \\
\text { protésico }\end{array}$ & $\begin{array}{l}\text { Desgaste de } \\
\text { resina de } \\
\text { recubrimiento }\end{array}$ \\
\hline Bergkvist & 2008 & ECC & $\begin{array}{l}\text { Sobrevida de la } \\
\text { carga inmediata. }\end{array}$ & - & $\bullet$ & $\bullet$ & $\bullet$ & - \\
\hline Agliardi & 2010 & ECC & $\begin{array}{l}\text { Sobrevida de la } \\
\text { carga inmediata. }\end{array}$ & - & - & - & - & - \\
\hline Eccellente & 2011 & ECC & $\begin{array}{l}\text { Efectividad de la } \\
\text { carga inmediata. }\end{array}$ & $\bullet$ & - & $\bullet$ & - & - \\
\hline Ganeles & 2001 & ECC & $\begin{array}{l}\text { Efectividad de la } \\
\text { carga inmediata. }\end{array}$ & - & - & - & - & - \\
\hline Malo & 2011 & ECC & $\begin{array}{l}\text { Sobrevida de la } \\
\text { carga inmediata. }\end{array}$ & $\bullet$ & - & $\bullet$ & - & $\bullet$ \\
\hline Piano & 2015 & ECC & $\begin{array}{l}\text { Efectividad de la } \\
\text { carga inmediata } \\
\text { con implantes } \\
\text { inclinados. }\end{array}$ & - & - & - & - & - \\
\hline Testori & 2007 & ECC & $\begin{array}{l}\text { Efectividad de la } \\
\text { carga inmediata } \\
\text { con implantes } \\
\text { inclinados. }\end{array}$ & $\bullet$ & - & - & - & - \\
\hline Acocella & 2011 & ECC & $\begin{array}{l}\text { Efectividad de la } \\
\text { carga inmediata. }\end{array}$ & $\bullet$ & $\bullet$ & $\bullet$ & - & $\bullet$ \\
\hline
\end{tabular}

$\because$ Presencia del fenómeno. - : Ausencia del fenómeno. 
a solicitar, momento y número. La primera toma va desde inmediatamente después de la carga inmediata hasta 32 meses después. Esto último está muy relacionado con el tiempo de duración que tenía destinado el estudio. Se puede observar que los exámenes imagenológicos más solicitados eran dos: radiografías periapicales y panorámicas (Tabla III).

Al momento de observar la presencia o ausencia de complicaciones protésicas en una PFTCl, la literatura no es muy específica en demostrar estadísticamente este fenómeno. No obstante, varios estudios en que se manifestó la fractura completa de la estructura protésica, concuerdan que esta es la complicación más grave (Tabla IV).
En otro estudio (Drago et al., 2011), 128 pacientes fueron sometidos a PFTCl, donde se dejó como provisorio una prótesis acrílica atornillada de arco completo. Las complicaciones de la prótesis provisoria se dividieron casi por igual en maxilar (19) y mandíbula (18). Maxilar superior tuvo un poco más de fracturas protésicas (15) que en mandíbula (11) (Tabla V).

De todos los estudios analizados, solo uno fue específico en la resolución de las complicaciones que se pudiesen presentar en una prótesis provisoria de arco completo posterior a la carga inmediata (Maló et al.) (Tabla VI).

Tabla V. Presencia del tipo de fractura en prótesis provisoria en una PFTCI

\begin{tabular}{ccccc}
\hline $\begin{array}{c}\text { Prótesis } \\
\text { provisoria }\end{array}$ & Total & $\begin{array}{c}\text { Fractura } \\
\text { Estructura } \\
\text { protésica }\end{array}$ & $\begin{array}{c}\text { Fractura dentaria } \\
\text { adhesiva }\end{array}$ & $\begin{array}{c}\text { Fractura dentaria } \\
\text { cohesiva }\end{array}$ \\
\hline Maxilar & 19 & 15 & 0 & 4 \\
Mandíbula & 18 & 11 & 0 & 7 \\
Total & 37 & 26 & 0 & 11 \\
\hline
\end{tabular}

PTFCI: Prótesis fija total con carga inmediata.

Tabla VI. Complicaciones protésicas y su resolución para PFTCI.

\begin{tabular}{lll}
\hline \multicolumn{1}{c}{ Complicación } & \multicolumn{1}{c}{ Resolución } \\
\hline Fractura completa de la estructura protésica. & 1. Reparación de prótesis. \\
& 2. Ajuste de la oclusión. \\
& $\begin{array}{l}\text { 3. Plano de relajación. } \\
\text { Aflojamiento del tornillo protésico. }\end{array}$ & 4. Recomendar al paciente de n o sobrecargar \\
& la prótesis. \\
Desgaste del material de la prótesis y del tornillo protésico. & 1. Apretar tornillo. \\
& 2. Ajuste de la oclusión. \\
& 1. Reemplazo del tornillo protésico. \\
& 2. Control de oclusión. \\
\hline
\end{tabular}

PFTCl: Prótesis fija total con carga inmediata.

\section{DISCUSIÓN}

Como podemos ver en la literatura (Ganeles et al., 2001; Testori et al., 2008; Bergkvist et al., 2009; Agliardi et al., 2010; Acocella et al., 2011; Eccellente et al., 2011; Maló et al.; Abdulaziz et al., 2013; Piano et al., 2016) no existe un consenso sobre el cuándo, la cantidad y con qué frecuencia se deben efectuar los controles clínicos posterior a una PFTCl. No obstante, en un estudio realizado por Ibañez et al. (2005), donde a 41 pacientes se les trato con PFTCI puesta en función a las 24 horas posterior a la cirugía, se observó un protocolo de control un poco más exhaustivo que el visto en la literatura. Aquí cada paciente fue controlado en la $1^{\circ}$, $2^{\circ}$ y $4^{\circ}$ semana. Posterior a eso, los controles se distendieron hacia el $3^{\circ}, 6^{\circ}$ y $12^{\circ}$ mes. 
Bergkvist et al. en su estudio cerró las chimeneas de forma temporal durante todos los controles efectuados durante el primer mes, para luego cerrarlas definitivamente durante el resto del tiempo. Esto parece ser aconsejable, ya que el acceso hacia los tornillos sería más accesible durante los primeros 30 días. Periodo en el cual se ha observado una pérdida de la estabilidad primaria no despreciable (Duvina et al., 2013).

En el caso de las indicaciones para el paciente sobre los cuidados inmediatos de una PFTCI la literatura es inconsistente, ya que es muy poco lo que habla de ella. (Acocella et al.; Eccellente et al.; Maló et al.; Abdulaziz et al., 2013; Piano et al.). No obstante, hay una indicación que es muy importante y debe ser mencionada en este estudio. Esto tiene relación a que no todos los pacientes y no todos los lechos anatómicos están indicados para dientes con carga inmediata. Es muy recomendable que los pacientes sean sometidos a un régimen de dieta blanda durante el periodo de cicatrización de 3 meses, para limitar las fuerzas funcionales mientras ocurre la oseointegración. Debido a que esto no puede ser fácilmente cumplido, el acatamiento de órdenes por parte del paciente es un factor importante para lograr el éxito. (Schnitman et al., 1990).

Sobre el cepillado dental y retiro de sutura, estos no deberían estar indicados hasta no antes de la segunda semana posterior a la carga inmediata. Ganeles et al. en su estudio utilizo enjuagues de clorhexidina al $0,12 \%$ por 3 semanas inmediatamente después de la carga inmediata. Si relacionamos esta indicación con lo anteriormente mencionado, enjuagues y un cepillado muy suave estaría indicado en las primeras 3 semanas posteriores a la carga.

Corbella et al. (2011) a un total de 61 pacientes los sometio a PFTCl tanto en maxilar como en mandibula, todo esto con el objetivo de evaluar los resultados de un protocolo de mantenimiento para implantes. Las indicaciones que se le dio a cada paciente sobre la técnica de cepillado fueron las siguientes:

1. Inmediatamente después de instalar la estructura provisoria el cepillado solo se debe restringir a la superficie de la prótesis.

2. Luego de dos semanas se utilizó un cepillo interdental pequeño y de cerdas suaves. Además una seda dental con esponja. El barrido mecánico se limitó solo a los cuellos de los implantes y la superficie apical de la prótesis.
3. Posterior a las dos semanas se tuvo que indicar un cepillo interdental más grueso, debido al aumento del tamaño del espacio entre la prótesis y el tejido perimplantar. Esto debido al proceso de cicatrización.

4. Al momento de cambiar la estructura protésica provisoria por una definitiva (3 a 4 meses) se tuvo que indicar nuevamente un cepillo más pequeño, puesto que el contacto de una prótesis definitiva con la mucosa comparada con un provisorio es mucho más íntimo.

En todas las investigaciones analizadas al parecer el tipo de radiografía y momento en el que se solicita parecen ser parámetros totalmente subjetivos. No obstante, se pueden ver tendencias. Como lo es que en la mayoría de las investigaciones solicitan un examen radiográfico inmediatamente después de la cirugía y a los 6 meses. Generalmente es un set de periapical o una panorámica (Ganeles et al.; Testori et al.; Bergkvist et al.; Agliardi et al.; Acocella et al.; Eccellente et al.; Maló et al.; Abdulaziz et al., 2013; Piano et al.). Tomar una radiografía panorámica inmediatamente después de la cirugía parece ser una excelente decisión, puesto que permite tener una idea generalizada de la situación de los implantes y la relación con la rehabilitación correspondiente. Pero si quisiéramos tener información radiológica más exacta, la radiografía periapical debería ser nuestra primera elección, ya que esta posee una distorsión mucho más baja que la de la radiografía panorámica, que corresponde a un $25 \%$ de magnificación.

Por otra parte, el tomar un set de periapicales inmediatamente después de la carga inmediata resulta muy útil. Esto se debe a que nos otorga una información base que sirve como punto de comparación para el futuro, como lo demuestra el estudio de Azcárate (Azcárate et al., 2001). Teniendo en cuenta que entre el día de la carga y tres meses después existe el mayor remodelado óseo (Duvina et al.) sería muy beneficioso tomar un set de periapical el día de la carga y otra 3 meses después. De esta forma, y efectuando la medición mencionada en el párrafo anterior, tendríamos una idea de cuánto hueso se ha modificado en este periodo que es el más crítico.

En la literatura se recomienda que la PFTCI no sea retirada durante el proceso de oseointegración, antes de 3 meses. Si algún implante presentara signos de infección, éste debería ser tratado de forma conservadora si fuera posible. Desmontar la prótesis provisional antes de tiempo puede interferir en la oseointegración del resto de los implantes (Concejo 
Cútoli \& Montesdeoca García, 2005). Esto concuerda con el estudio de Duvina et al., donde se observa que la estabilidad inicial del implante desde el día de la carga hasta el $1^{\circ}$ mes decae bruscamente para luego reposicionarse a los 3 meses. Esto indicaría que el momento más seguro para sacar la prótesis provisoria e instalar la definitiva seria entre el $3^{\circ}$ y $4^{\circ}$ mes post carga. Procedimiento que en la mayoría de las investigaciones se tiende a seguir (Ganeles et al.; Testori et al.; Bergkvist et al.; Agliardi et al.; Acocella et al.; Eccellente et al.; Maló et al.; Abdulaziz et al., 2013; Piano et al.).

Como se puede observar en esta revisión bibliográfica de tipo narrativa, todas las investigaciones recopiladas son ensayos clínicos controlados, conocidos por el gran valor científico que entregan. No obstante, ninguno de ellos otorga información concisa y objetiva sobre cómo se debería efectuar un control clínico para una PFTCI.

La distintas complicaciones que se hacen presente posterior a la instalación de una PFTCI (Tabla IV, $\mathrm{V} \mathrm{y} \mathrm{VI)} \mathrm{indican} \mathrm{que} \mathrm{es} \mathrm{necesario} \mathrm{estandarizar} \mathrm{el}$ número y momento en que se deben citar los pacientes a control, los parámetros de elección para la toma de radiografía, y el momento indicado para el retiro de la prótesis provisoria e instalación de la definitiva. Estas acciones, que son de primera orden, constituyen un incentivo para la confección de un protocolo clínico para una PFTCI (Anexo). De esta manera cualquier eventualidad o complicación posterior al tratamiento puede terminar en una resolución exitosa y benéfica para el paciente.

\section{CONCLUSIONES}

No existe evidencia científica que determine cuales son los procedimientos clínicos post-operatorios para el control quirúrgico y protésicos en pacientes rehabilitados mediante prótesis fija total con carga inmediata, ya que en la literatura no hay estudios relacionados sobre el tema.

La presencia de complicaciones posterior a la instalación de una prótesis fija total con carga inmediata hace necesaria la confección de un protocolo de control clínico para asegurar un tratamiento exitoso y benéfico para el paciente. La complicación más grave es la fractura total de la estructura protésica y la causa más común es la sobrecarga oclusal.
No existe evidencia concreta sobre el número, momento y frecuencia de sesiones necesarias para el control clínico de una PFTCI. No obstante, es aconsejable llevar un control minusioso entre el día de la carga inmediata y el $4^{\circ}$ mes. Esto debido al intenso proceso de remodelación ósea.

El momento más indicado para la extracción de la prótesis provisoria por primera vez e instalación de la prótesis fija total definitiva es durante el $3^{\circ}$ y $4^{\circ}$ mes posterior a la carga inmediata. Puesto a que en ese momento la estabilidad del implante ya es segura.

La evidencia científica sobre el número, momento y tipo de examen imagenológico a solicitar es escasa e inconsistente. Sin embargo, solicitar un set de radiografías periapical el día de la carga y 3 meses después es muy ventajoso. Esto se debe a que entrega información relacionada con la integridad de las estructuras protésicas junto con la implantaría el día de la carga, y como el intenso remodelado ósea se desenvuelve alrededor de los implantes.

Es aconsejable llevar a cabo nuevas investigaciones que aporten de forma objetiva y concreta conocimiento sobre el correcto control clínico de prótesis fijas totales con carga inmediata.

FIGUEROA, A. E. \& RABY, O. I. Clinical control of patients treated with an immediately loaded implant-supported prosthesis. A systematic review. Int. J. Odontostomat., 12(1):21-28, 2018

ABSTRACT: There are many complications that can occur following placement with an immediately loaded implant-supported prosthesis in rehabilitation, these may generate damage to the provisional structure and even loss of the osseointegration from the dental implants. Limited evidence about clinical behavior post-installation, creates the need to better assess possible future complications that may arise. In this review, a systematic research was made in the PubMed database. The filters used were "immediate loading", "full arch" and "Resonance frequency analysis". The results were subject to the inclusion and exclusion criteria. A total of 15 studies were selected. All of the studies were randomized clinical trials, many complications were recorded, such as fractures, dislodgement and wear of the prosthesis structure. It was observed that from the first day of immediate loading until the first month, Implant Stability Quotient value decreases, subsequently, progressively increasing. There is no evidence about the number and range of sessions needed to assess the clinical control of a immediate loading fixed rehabilitation. The ideal time for removal of the provisional prosthesis and installation of the restorative rehabilitation, is during the third and fourth month after the 
immediate loading. Limited evidence was found regarding the number, the time and type of radiological images for clinical support. The possibility of several complications after an installation of a immediately loaded implant-supported prosthesis are very real, therefore is necessary to establish a clinical protocol for controls regarding this treatment.

KEY WORDS: immediate loading, full arch, resonance frequency analysis.

\section{REFERENCIAS BIBLIOGRÁFICAS}

Acocella, A.; Ercoli, C.; Geminiani, A.; Feng, C.; Billi, M.; Acocella, G.; Giannini, D. \& Sacco, R. Clinical evaluation of immediate loading of electroeroded screw-retained titanium fixed prostheses supported by tilted implant: a multicenter retrospective study. Clin. Implant Dent. Relat. Res., 14 Suppl. 1:e98-108, 2011.

Agliardi, E.; Clericò, M.; Ciancio, P. \& Massironi, D. Immediate loading of full-arch fixed prostheses supported by axial and tilted implants for the treatment of edentulous atrophic mandibles. Quintessence Int., 41(4):285-93, 2010

Bergkvist, G.; Nilner, K.; Sahlholm, S.; Karlsson, U. \& Lindh, C. Immediate loading of implants in the edentulous maxilla: use of an interim fixed prosthesis followed by a permanent fixed prosthesis: a 32-month prospective radiological and clinical study. Clin. Implant Dent. Relat. Res., 11(1):1-10, 2009.

Concejo Cútoli, C. \& Montesdeoca García, N. Carga inmediata en implantes dentales. Rev. Esp. Cirug. Oral Maxilofac., 27(5):25569, 2005.

Corbella, S.; Del Fabbro, M.; Taschieri, S.; De Siena, F. \& Francetti, L. Clinical evaluation of an implant maintenance protocol for the prevention of peri-implant diseases in patients treated with immediately loaded full-arch rehabilitations. Int. J. Dent. Hyg., 9(3):216-22, 2011.

Drago, C.; del Castillo, R. \& Peterson, T. Immediate occlusal loading in edentulous jaws, CT-guided surgery and fixed provisional prosthesis: a maxillary arch clinical report. J. Prosthodont., 20(3):209-17, 2011.

Duvina, M.; Barbato, L.; Buti J.; Delle Rose, G.; Brancato, L.; Casella, G.; Longoni, S.; Sartori, M.; Amunni, F. \& Tonelli, P. Immediate loading with LASER-treated surface implant: two-year resonance frequency analysis follow-up. J. Craniofac. Surg., 24(3):849-55, 2013.

Degidi, M.; Piattelli, A.; Felice, P. \& Carinci, F. Immediate functional loading of edentulous maxilla: a 5-year retrospective study of 388 titanium implants. J. Periodontol., 76(6):1016-24, 2005.

Eccellente, T.; Piombino, M.; Piattelli, A.; D'Alimonte, E.; Perrotti, V. \& lezzi, G. Immediate loading of dental implants in the edentulous maxilla. Quintessence Int., 42(4):281-9, 2011.

Esposito, M.; Grusovin, M. G.; Maghaireh, H. \& Worthington., H. V. Interventions for replacing missing teeth: different times for loading dental implants. Cochrane Database Syst. Rev., (3):CD003878, 2013.

Ibañez, J. C.; Tahhan, M. J.; Zamar, J. A.; Menendez, A. B.; Juaneda, A. M.; Zamar, N. J. \& Monqaut, J. L. Immediate occlusal loading of double acid-etched surface titanium implants in 41 consecutive full-arch cases in the mandible and maxilla: 6- to 74-month results. J. Periodontol., 76(11):1972-81, 2005.

Ganeles, J.; Rosenberg, M. M.; Holt, R. L. \& Reichman, L. H. Immediate loading of implants with fixed restorations in the completely edentulous mandible: report of 27 patients from a private practice. Int. J. Oral Maxillofac. Implants, 16(3):418-26, 2001.
Kinsel, R. P. \& Liss, M. Retrospective analysis of 56 edentulous dental arches restored with 344 single-stage implants using an immediate loading fixed provisional protocol: statistical predictors of implant failure. Int. J. Oral Maxillofac. Implants, 22(5):823-30, 2007.

Grunder, U. Immediate functional loading of immediate implants in edentulous arches: two-year results. Int. J. Periodontics Restorative Dent., 21(6):545-51, 2001.

Horwitz, J.; Zuabi, O.; Peled, M. \& Machtei, E. E. Immediate and delayed restoration of dental implants in periodontally susceptible patients: 1-year results. Int. J. Oral Maxillofac. Implants, 22(3):4239, 2007.

Maló, P.; de Araújo Nobre, M.; Lopes, A.; Francischone, C. \& Rigolizzo, M. "All-on-4" immediate-function concept for completely edentulous maxillae: a clinical report on the medium (3 years) and long-term (5 years) outcomes. Clin. Implant Dent. Relat. Res., 14 Suppl. 1:e139-50, 2012

Peñarrocha-Oltra, D.; Covani, U.; Peñarrocha-Diago, M. \& PeñarrochaDiago, M. Immediate loading with fixed full-arch prostheses in the maxilla: review of the literature. Med. Oral Patol. Oral Cir. Bucal, 19(5):e512-7, 2014

Piano, S.; Romeo, E.; Sbricoli, L.; Pisoni, G.; Cea, N. \& Lops, D. Simplified procedure for the immediate loading of a complete fixed prosthesis supported by four implants in the maxillary jaw: a 2year prospective study. Clin. Oral Implants Res., 27(12):e154-e160, 2016.

Pieri, F.; Aldini, N. N.; Fini, M. \& Corinaldesi, G. Immediate occlusal loading of immediately placed implants supporting fixed restorations in completely edentulous arches: a 1-year prospective pilot study. J. Periodontol., 80(3):411-21, 2009.

Sanz, M.; Ivanoff, C. J.; Weingart, D.; Wiltfang, J.; Gahlert, M.; Cordaro, L.; Ganeles, J.; Bragger, U.; Jackowski, J.; Martin, W. C.; Jung, R. E.; Chen, S. \& Hammerle, C. Clinical and radiologic outcomes after submerged and transmucosal implant placement with two-piece implants in the anterior maxilla and mandible: 3-year results of a randomized controlled clinical trial. Clin. Implant Dent. Relat. Res., 17(2):234-46, 2015

Testori, T.; Del Fabbro, M.; Capelli, M.; Zuffetti, F.; Francetti, L. \& Weinstein, R. L. Immediate occlusal loading and tilted implants for the rehabilitation of the atrophic edentulous maxilla: 1-year interim results of a multicenter prospective study. Clin. Oral Implants Res., 19(3):227-32, 2008.

van Steenberghe, D.; Glauser, R.; Blombäck, U.; Andersson, M.; Schutyser, F.; Pettersson, A. \& Wendelhag, I. A computed tomographic scan-derived customized surgical template and fixed prosthesis for flapless surgery and immediate loading of implants in fully edentulous maxillae: a prospective multicenter study. Clin. Implant Dent. Relat. Res., 7 Suppl. 1:S111-20, 2005.

Weber, H. P.; Morton, D.; Gallucci, G. O.; Roccuzzo, M.; Cordaro, L. \& Grutter, L. Consensus statements and recommended clinical procedures regarding loading protocols. Int. J. Oral Maxillofac. Implants, 24 Suppl::180-3, 2009.

Dirección para correspondencia:

Emanuel Figueroa Ahumada

Cirujano Dentista

Universidad de Valparaíso

Valparaíso - CHILE

Email: emanuelfiguero@hotmail.com

Recibido : 06-09-2017

Aceptado: 06-01-2018 